\title{
In vivo Studies for Drug Development via Oral Delivery: Challenges, Animal Models and Techniques
}

\author{
Katherine Brake ${ }^{1}$, Ashwini Gumireddy ${ }^{2}$, Amit Tiwari ${ }^{3}$, Harsh Chauhan² and Dunesh Kumari ${ }^{1}$ \\ ${ }^{1}$ Chemistry Department, College of Saint Mary, Omaha, NE \\ ${ }^{2}$ School of Pharmacy and Health Professions, Creighton University, Omaha, NE \\ ${ }^{3}$ Department of Pharmacology and Experimental Therapeutics, University of Toledo, Toledo, $\mathrm{OH}$
}

\begin{abstract}
Drug discovery and development involves a complex iterative process of new molecules synthesis, formulations, and in vitro analysis, followed by biochemical and cellular assays, with final validation in animal models, and ultimately in humans. The purpose of this article is to present a concise overview of the rationale and challenges for performing in vivo studies, the types of animal models, and modern in vivo research techniques for oral drug delivery.
\end{abstract}

Keywords: Drug discovery; in vivo; in vitro; Animal models; Drug development; Biological barriers; Gastrointestinal tract

\section{Introduction}

The process of drug discovery and development takes an average of 10 to 15 yrs and (depending on the therapeutic area) as much as 1 billion USD to bring a single drug into market [1-4]. New drugs are approved by the Food and Drug Administration (FDA), before it may be legally introduced into the market [2]. FDA is the first agency structured through Title 21 of the U.S. code of federal regulations established in 1906 to regulate the quality of food and medicine [5]. Although every disease and every scientific approach have their exactitudes, drug discovery and development process itself is nowadays conducted in relatively standardized sequence of discovery and development phases. They are driven by regulatory requirements and the aim to avoid the unnecessary cost by early elimination of unlikely drug candidates [6]. A schematic representation of the process for new drug development and its timeline is shown in Figure 1 The timeline breaks down into 5 main categories (Drug Discovery, Preclinical, Clinical Trials, FDA Review, and FDA Approved Drug) with three subcategories within Clinical Trials [7]. The mechanism for drug discovery alone takes an average of six years with initially hundreds and thousands of molecules screened before the preclinical stage can begin. Drug discovery begins with target selection and lead discovery which can progress into identifying the characteristics of possible medicines ascertained by preclinical trials (Table 1, Figure 2) i.e. The new drug is biologically characterized for pharmacologic and toxicologic effects and for potential therapeutic applications. The preclinical stage encompasses the use of in vitro and in vivo studies to develop a drug that can safely and effectively be administered for clinical trials. These clinical trials are broken up into three phases to allow the study of the safety and pharmacology, the effectiveness of a compound in human trials, and to confirm previous findings in a larger population [8-10]. The process results in more failures than successes with approximately only $10 \%$ of the molecules entering the clinical trials. Upon FDA review, the drug will either be approved or rejected. If a drug is rejected, the applicant is given reasons why and what information could be provided to improve the submission and allow the application to be acceptable [9].

After identification and characterization of the new drug, determination and validation of the reasonable biological response testing the pharmacokinetics parameters (drug molecule absorption, distribution, metabolism and elimination) is one of the most important steps during drug discovery and development. Validation techniques ranges from in vitro testing i.e. testing done on working cells and or tissues removed form living organisms to in vivo testing i.e. sampling from laboratory animals. In vitro studies allow a drug to be tested at a minimized cost, ensuring to "fail fast, but fail cheap" if the drug does not perform as anticipated $[11,12]$. In addition to reducing cost, in vitro studies are not performed on living organisms, which also reduces ethical dilemmas and experimental restriction. These cost effective and morally upstanding studies provide a basis for the predictably of a drug's success or failure in in vivo studies and later, clinical studies. In vivo studies are essential to drug development because they provide the ability to evaluate a drug's characteristics, including physiological and biochemical processes, such as adverse effects and drug-drug interactions that cannot be observed in vitro. Many challenges surrounding in vivo studies can be overcome by choosing an animal model that best illustrates the human system the drug will affect. A correct animal model acknowledges the bioavailability in the gastrointestinal tract due to $\mathrm{pH}$ or fasted/fed state and can mimic potential reticuloendothelial system (RES) uptake. New techniques for in vivo methods have conceded to less invasive applications for collecting data crucial to metabolism and drug-drug interactions before FDA approval. The purpose of this paper is to present the fundamental need and role of in vivo studies in drug development.

\section{Comparision of in vitro and in vivo Research}

The evaluation of efficacy and safety of a new drug candidate includes in vitro and in vivo studies that can be carried out throughout all stages of drug development. The proper selection and applications of correct models, as well as appropriate data interpretation are essential to provide the basic knowledge about the drug pharmacodynamics and are critically important in decision making and successful advancement of drug candidates for clinical trials. The use of in vivo studies prior to a drug becoming commercially available is crucial for understanding the characteristics and effects of a drug within a living organism. In vitro studies focus on major influences that may affect the drug release in vivo.

*Corresponding author: Dunesh Kumari, Chemistry Department, College of Sain Mary, Omaha, NE, Tel: (402)-399-2416; E-mail: DKumari@csm.edu

Received July 24, 2017; Accepted September 05, 2017; Published September 11,2017

Citation: Brake K, Gumireddy A, Tiwari A, Chauhan H, Kumari D (2017) In vivo Studies for Drug Development via Oral Delivery: Challenges, Animal Models and Techniques. Pharm Anal Acta 8: 560. doi: 10.4172/2153-2435.1000560

Copyright: (c) 2017 Brake K, et al. This is an open-access article distributed under the terms of the Creative Commons Attribution License, which permits unrestricted use, distribution, and reproduction in any medium, provided the original author and source are credited. 


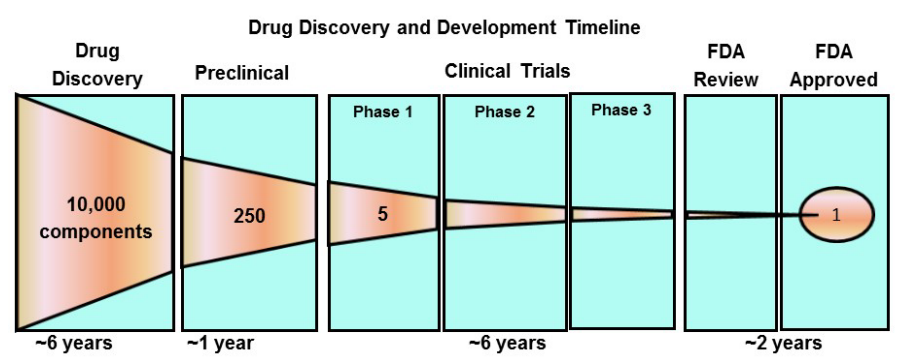

Figure 1: The approximate timeline for drug discovery and development with an FDA approved product.

\begin{tabular}{|c|c|c|c|}
\hline Objective & Stage number & $\begin{array}{c}\text { Description } \\
\text { Average number of components } \\
\text { entering each stage }\end{array}$ & $\begin{array}{c}\text { Average of } \\
\text { animals }\end{array}$ \\
\hline $\begin{array}{c}\text { Discovery and selection of potential new } \\
\text { medicines }\end{array}$ & 1 & Target identification & $1,000,000$ \\
\cline { 2 - 4 } & 2 & Ldentification of possible medicines & 1,000 \\
\hline $\begin{array}{c}\text { The characterization of promising } \\
\text { candidate medicine }\end{array}$ & 3 & Lead identification & 200 \\
\hline Ensuring the safety of selected \\
candidates
\end{tabular}

Table 1: Animals are used in all steps of drug development and discovery, excluding clinical studies on humans. In vitro studies are used to determine dissolution and drug release rates before in vivo studies to allow successes and failures to be done at a minimized cost with less ethical restrictions. Once in vivo studies begin, components lessen with each advancing stage until human clinical trials are obtainable. Animal study data is used in all steps of drug discovery and development with the exception of clinical studies on humans. In vivo studies are heavily used during the characterization of new medicines [68].

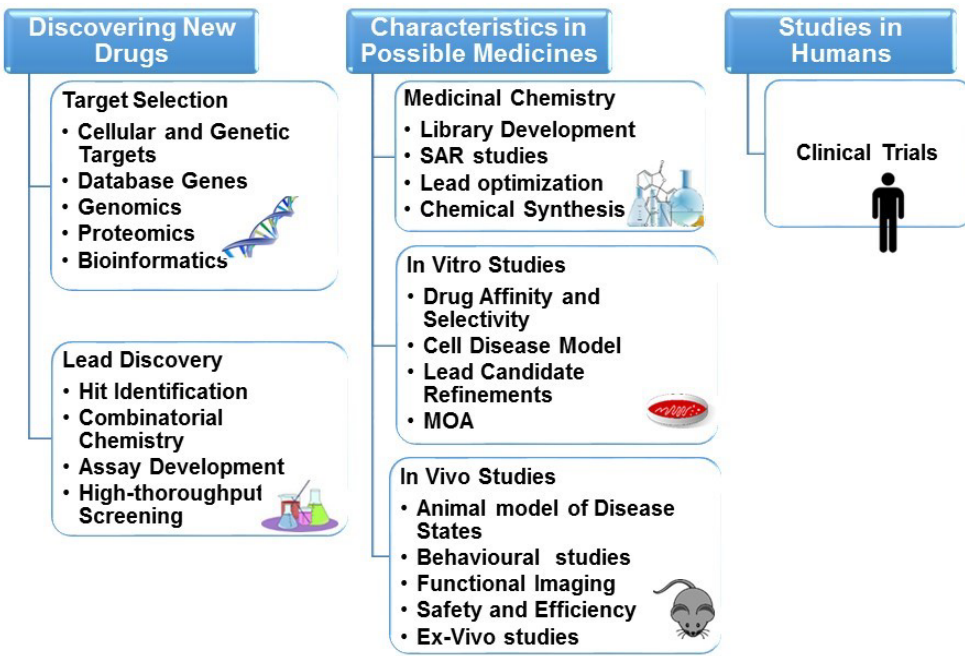

Figure 2: The process of drug development begins with the discovery of new drugs, which includes target selection and lead discovery. After lead discovery, characteristics in possible medicines are identified through medicinal chemistry, in vitro studies, and in vivo studies. This allows clinical trials in humans to take place, eventually resulting in an FDA approved product (FDA, food and drug administration).

\section{In vitro research}

As previously stated, in vitro studies are critical to the drug development process due to their ability to provide a basis for predicting the clinical results of a drug, including the success or failure of the drug in vivo [13]. Usually, in vitro analyses are performed in the early stages of the drug discovery process, when the selectivity and possible interactions of the candidate drug towards the desired therapeutic target are established [14]. In vitro studies first focus on drug absorption, distribution, metabolism, and excretion which allows a more direct assessment of drug performance [15]. This is achieved through the study of drug dispersion, absorption/dissolution and permeability using samples of tissue, cell, or bacteria outside of their biological systems. Through these in vitro studies, the activity of the candidate drug upon different features, such as the induction of cell death and proliferation, changes in gene expression, changes in the protein profile, biochemical dosage of mediators, changes in cell cycle assessment, multidrug resistance potential, and others is observed [14]. Nowadays, in vitro pharmacological profiling including the absorption, distribution, metabolism, excretion and toxicity studies are is increasingly being used earlier in the drug discovery process to identify undesirable off-target activity profiles that could hinder or halt 
the development of candidate drugs or even lead to market withdrawal if discovered after a drug is approved [16,17]. According to the National Research (US) Committee, "In vitro methods are usually the methods of choice for large-scale production by the pharmaceutical industry because of the ease of culture for production, compared with use of animals, and because of economic considerations" [18]. It is easier to provide or reproduce isolated cells or tissue cells using polymerase chain reaction (PCR) as well as keep the cost of sample lower than live samples, such as those used for in vivo. Some of the unspoken advantages of using in vitro include avoiding the need to submit animal protocols to IACUCs in addition to avoiding or decreasing the need for laboratory personnel experienced in animal handling [19].

Although, this has a positive impact on the development of a drug because successes and failures in vitro are done at a minimized cost and dissuade any ethical restrictions [15]. Since in vitro studies cannot entirely predict the influence the drug will have on organs and organ systems, or even the interaction with other drugs, in vivo studies are needed to clarify data concerning therapeutic drugs before clinical trials are carried out. In vivo studies allow the long-term effects of the drug to be monitored and observed, as well as determining the bioequivalency, safety, dosing regimen, positive and adverse effects, and the drugdrug interactions in a living system. If an in vitro/in vivo correlation is observed, a bioequivalency study may be waived which allows time and costs to be reduced [20]. Results from in vitro/in vivo correlation (IVIVC) studies have been used to select the appropriate excipients and optimize the manufacturing processes for quality control purposes, and for characterizing the release patterns of newly formulated immediate release, and modified-release products relative to the references which also allows reduction in overall costs [21].

\section{The need for in vivo research}

In vitro studies provide relevant information on the mechanism of action of drugs which is useful for making hazard-based decisions and informing decision-making in drug development process, but without linking the in vitro toxicodynamic measurements to in vivo toxicokinetics, the relevance to human exposure scenarios and risk assessment is limited [22]. There are limitations for the study of drug dispersion and permeability in vitro and the inability for in vitro studies to accurately mimic a live biological system which produces the need for in vivo studies. In vitro data cannot entirely predict the interaction of organs and organ systems with the drug or the drug's interaction with other drugs [23]. In vitro dissolution has been recognized as an important element in drug development, however, it does not possess the ability to provide a quantitative interpretation of absorption, distribution, metabolism, and excretion in animal and human models [24]. For substances with a poor aqueous solubility and for which solubility is the major limitation of drug absorption, in vitro dissolution media reflecting the in vivo conditions are crucial for the rapid screening and assessment of formulations [25]. The in vitro method of dissolution testing can characterize how an active pharmaceutical ingredient is extracted out of a solid dosage form and can indicate the efficiency of in vivo dissolution, but does not provide any information on drug substance absorption or drug-drug interactions within a system [26]. It is still unclear on how in vitro assays concerning concentration relate to dosage and exposure patterns that animals or humans would experience during in vivo testing when exposed to natural situations. There is also difficulty in determining exactly how much of the chemical used in the in vitro model has reached the site of action. An understanding of both internal and external exposure is necessary to put the in vitro results into context and avoid misinterpretation of the data and its relevance to toxicity endpoints [22].
In vitro/in vivo correlation, as mentioned previously, is not only important in waiving bioequivalency studies, but more commonly used in drug development to assist in quality control for certain scaleup and post-approval changes, to ensure safety, and is increasingly becoming an integral part of extended release drug development [27]. There have been attempts to establish IVIVC for a variety of drugs, such as aspirin, that initially reported a poor IVIVC, but after several adjustments to methodology and advancements in technology, the drug that is widely used today has reported a good IVIVC, therefore ensuring it's safety and effectiveness [28,29]. Even with promising in vitro studies, there is not a guarantee for success with in vivo studies. In the case of metabolic tumors, in vitro studies could not be translated in vivo at preclinical stage and beyond due to a variety of factors, such as limitations to mimic the micro- and macro- environment [30]. In vivo testing may even be needed to distinguish discrepancies between two separate in vitro study results. In a study regarding the antitumor activity of amidino-substituted benzimidazole and benzimidazo [1,2-a] quinoline derivatives, the $2 \mathrm{D}$ cell cultures used for in vitro testing were found comparable to $3 \mathrm{D}$ cell cultures, but significant disagreements in data indicated false positive results, in which in vivo profiling was needed for confirmation [31]. This strengthens the notion that in vitro studies alone, no matter how promising, are not a reliable indicator of a drug's performance which renders the necessity of in vivo studies.

In addition to drug dispersion and permeability, in vitro studies are also used to study toxicological effects of drugs. While there are many benefits to in vitro toxicology studies, such as having high throughput, ability to use low quantities, and reduce animal testing, it is still not practical to set up screens for all possible toxic effects that may be observed in animals and humans [32].

All of the factors that pertain to the influence of drug dissolution and release cannot simply be present in in vitro experiments [33]. Furthermore, the cell use for an in vitro study does not allow longterm effects to be known due to inadequate preservation [34]. Due to the lack of capacity to determine the bioequivalence, the safety, the dosage regimen, the drug effects, adverse effects, and drug-drug interactions in an intact, living system with in vitro studies, in vivo research is necessary to establish a drug that is safe and effective in humans by continuing work with drugs that are shown to be the most promising from in vitro research and acknowledging the drugs that might have cause for concern from the in vitro data. Overall, in vitro models have limited values, as they reflect only one particular aspect of the whole picture, whereas the in vivo results are multifactorial, provide the combined effect of drug permeability, distribution, metabolism and execration, and can yield a measurable set of pharmacokinetics parameters and toxicological endpoints. Regardless of thoroughness and completeness of the in vitro work, animal studies are required to measure drug exposures and to determine potential toxicities [22].

\section{Challenges of in vivo research}

Lead drugs (chemicals or biologics like peptides, antibodies, and vaccines etc.) are administered into the body (animal or human) via intravenous delivery (vascular endothelial lining, particularly for tumor vasculature and blood brain barrier targeting), oral administration (gastrointestinal lining), and upper airway administration (pulmonary epithelium). The delivery of the lead drugs to the target sites frequently involves various biological barriers, they have to cross different specialized epithelia, either lung or gastrointestinal (GI) tract epithelia, to reach the blood compartment, tumoral vascular endothelium, or the blood brain barrier (BBB) to access pathological tissues via the 
blood circulation [35]. These barriers have exceptional effects on drug bioavailability/potential therapeutic application of the drug that present themselves in studies involving whole organisms that are not readily available and cannot be replicated satisfactorily employing in vitro studies.

Oral drug delivery is the most widely used and most readily accepted form of drug administration as it is simple, painless and selfadministered [36,37]. It is the most convenient and safe administration route, particularly for chronic delivery, but it poses a number of challenges for the formulator in terms of bioavailability (fraction of drug actually reaching the circulation) due to degradation by enzymes and harsh $\mathrm{pH}$ conditions, low solubility of some drugs or limited absorption by the GIT epithelium [38]. Poor solubility, stability, and bioavailability of many drugs make achieving therapeutic levels via the gastrointestinal (GI) tract challenging [39]. Physiological factors that effects in vivo performance of the drug dosage form in the respective preclinical models are still insufficiently understood; these include GI condition, mechanical stress, effects of food, enzymatic or $\mathrm{pH}$ related degradation of drug and its excipients, drug dosage release profile and absorption in various GI segments, and the direct influence of some excipients on drug metabolism and transport [35]. Most of these factors have little or no impact for highly soluble drugs but are of greater influence for slightly or poorly soluble drugs, since their in vivo performance relies to a greater extent on the characteristics of their dosage form [35]. The two major in vivo challenges discussed in this review include influence of local physiological condition of the GI tract on in vivo performance of the drug dosage form and the biological barriers associated with drug delivery. These influences and factors may impact the pharmacokinetic properties of a drug in a fashion that was not foreseeable by in vitro experiments.

Gastrointestinal challenges: The GastroIntestinal (GI) tract is a hostile environment for biomacromolecules because it is optimized to break down nutrients and deactivate pathogens. The acidic $\mathrm{pH}$ in the stomach results in the protonation of proteins, leading to their unfolding which exposes more motifs that are recognized by protein-degrading enzymes such as pepsin in the stomach and chymotrypsin in the small intestine [40]. These enzymes, along with others, cleave proteins and nucleic acids into smaller particles. Once these fragments have reached the colon, enzymatic processes further degrade the biomacromolecules. Differences between a fasted or fed state ( $\mathrm{pH}$, surfactants, movement, enzymes, and ionic strength) and the inhibitory effect ingested substances may have on synergizing or antagonizing a drug are two ways in which dietary substances can affect the degradation processes and therefore, the bioavailability of a drug. These factors influence the bioavailability of the drug and these factors may manipulate the pharmacokinetic properties of the drug and can only be monitored through in vivo studies. Presently, there is no single in vitro system that has been developed to simulate the sequential use of enzymes in physiological amounts, the physiological working $\mathrm{pH}$ of endogenous enzymes, excretion of digestive products, appropriate mixing at each step of digestion, the times spent at each step of digestion, and peristalsis, each of which being different during the fasted or fed state [33]. To ensure that a drug will be successful in terms of bioavailability, various in vitro studies are carried out to accomplish the task set forth for the specific drug before continuing with in vivo studies. The $\mathrm{pH}$ is one of the most important factors that depends on the fasted or fed state of the stomach; the dissolution, solubilization, and absorption of a drug is considerably affected by $\mathrm{pH}$ [41]. The human stomach has a dynamic $\mathrm{pH}$ that varies depending on the state of the stomach; the $\mathrm{pH}$ greatly increases in its basicity in the fed state, which changes the drug release characteristics. This occurs, in part, due to the complexity of processes which take place in the GI tract and in part to the complex pharmacokinetics of drugs. Food in general influences the $\mathrm{pH}$, but the type of food also can affect the metabolism of some drugs or enterohepatic recirculation, e.g. by inhibiting hydrolytic enzymes produced by intestinal bacteria. Metabolism-related fooddrug interactions are highly dependent on the composition of the food, namely, they are mostly associated with fruits, vegetables, alcoholic beverages, teas and herbs. For example, ingestion of grapefruit juice can enhance systemic exposure of a drug metabolized by CYP3A4 by $1400 \%$ [42]. Also, ingestion of carbohydrates can reduce the oxidation of drugs, such as antipyrine and theophylline [43]. Also, ingestion of grapefruit juice can enhance systemic exposure of a drug metabolized by CYP3A 4 by $1400 \%$ [42]. The gastrointestinal effects due to a fasted or fed system should be investigated considering the potential effect on the pharmacokinetic properties of a drug.

The maneuverability of the gastrointestinal tract also influences drug absorption by changing the effective surface for absorption. There are four notable phases during the fasted state, three of which include gastric contractions, and the last phase is expulsion of non-digested materials [41]. The stomach spends most of its time varying from two phases in the fed state, switching from high-intensity contractions to expulsion during the fed state [41]. In the small bowel, muscle contractions occur irregularly, varying in strength and type due to the nutrients absorbed from the food. The time a substance, particularly a drug, spends in the GI tract varies depending on the state as well as its relation to nutrient absorption. Sjogren et al. has summarized the biopharmaceutical factors influencing the in vivo drug performance (pharmacokinetics/and or dynamic) in respective preclinical models like pig, dog, mouse, rat and also in human [35]. In vitro testing of significant barriers to intestinal absorption in humans, specifically permeability and solubility of a drug, are recommended in the early assessment of colonic absorption [44]. The lack of successful attempts to predict gastrointestinal absorption of poorly soluble drugs creates the need for a better understanding of the in vivo GI process, which includes the changing physiological conditions, the fed versus fasted state, and the effect of pharmaceutical product characteristics. In vivo studies that focus on these aspects play a crucial role in understanding the pharmacokinetics of an orally administered drug, necessitating a more rational approach to develop reliable in vitro/in vivo correlations and better methods for predicting the rate of GI absorption and potential bioavailability for drugs that are administered orally [45].

Biologics barriers for oral drug delivery: The delivery of the lead drugs to the target sites frequently involves various biological barriers, they have to cross different specialized epithelia, either lung or gastrointestinal (GI) tract epithelia, to reach the blood compartment, vascular endotheliu, or the blood brain barrier (BBB) to access pathological tissues via the blood circulation. This is not an easy task, even for nanometric objects ( $1 \mathrm{~nm}$ to $1000 \mathrm{~nm}$ ), and available pathways are limited to epithelium porosity or transcytosis routes [38]. The intestinal mucosa barrier limits oral drug delivery into the systemic circulation, consequently preventing the drug from reaching the target tissue. If the target organ in in the central nervous system (CNS), the drug has to cross the $\mathrm{BBB}$. The $\mathrm{BBB}$ helps to maintain a separate pool of neuroactive agents between the CNS and the peripheral tissues, which prevents signal crosstalk. The BBB can be an obstacle in developing therapeutic agents to treat brain diseases like brain tumors, Alzheimer's and Parkinson's diseases [46]. 
The intestinal mucosa barrier is formed by an epithelial cell layer which mainly consists of absorptive enterocytes and mucus producing Goblet cells, endocrine and Paneth cells spread along the epithelium. The GI tract epithelium is covered by a layer of mucus. Immunocompetent cells, such as B and T lymphocytes and dendritic cells are located beneath the epithelium. The small intestine wall possesses a rich blood network, and the GI tract blood circulation is nearly a third of cardiac output flow, underlining the importance of exchanges between the GI tract lumen and the blood circulation [38]. The drug that is absorbed through the intestinal mucosa barrier will be transported by the blood vessels throughout the body, including the brain, if the drug can cross the BBB. The BBB is a highly specialized structural, transport and biochemical (enzymatic) barrier, that mainly consists of microvascular endothelial cells. It regulates the entry of compounds and cells between blood and brain and, thus, has a fundamental role in brain homeostasis. Much of the structural barrier is due to the presence of tight junctions between the cerebral endothelial cells that limit paracellular diffusion [47].

Both the intestinal mucosa and BBB have enriched peptidases and other metabolism enzymes (e.g., cytochrome P450) that degrade small and macro molecules before or during passage through these barriers. The efflux pumps (e.g., multidrug-resistant related proteins (MRP), P-glycoproteins (Pgp), and breast cancer-related proteins (BCRP)) can expel a wide variety of drug molecules from the membranes to prevent them from crossing through the transcellular pathway [46].

The reticuloendothelial system (RES) is comprised of various tissues and organs, including the spleen, the Kupffer cells of the liver, and various lymphatic tissues [48]. The RES functions to increase drug clearance by phagocytizing foreign opsonized materials, clearing the drug rapidly within seconds and eliminating the drug's therapeutic effects $[49,50]$. Therefore, the RES, Intestinal mucous and $\mathrm{BBB}$ constitute a major obstacle and sink to the efficient targeting of therapeutic agent in in vivo studies.

Nanoparticle drug delivery has emerged as potentially suitable approaches for overcoming pharmacokinetic limitations associated with traditional drug formulations. Several nanoparticles/nanocarriers like liposomes, polymer particles, micelles, dendrimers, quantum dots, and carbon nanotubes have been synthesized and tested for their therapeutic application [51]. They have proven advantageous at providing protection to therapeutic agents while efficiently delivering them into through the BBB in neurodegenerative/ischemic disorders and target relevant regions in the brain for regenerative processes, and prolonging the circulation lifetime of drugs. Nevertheless, as they enter the blood stream; certain nanoparticles are engulfed and eliminated by immune cells in the bloodstream (such as monocytes, platelets, leukocytes, and dendritic cells) and in tissues (such as resident phagocytes), thus limiting the site-specific bioavailability and preventing the success of outcomes [51,52]. Nanoparticles, like other colloidal carriers after intravenous administration, are normally retained mainly by the Kupffer cells of liver and macrophages of the spleen [53]. Under normal healthy conditions, the vascular endothelium is generally impermeable to nanoparticles. Places that exhibit "leaky vasculature," as is the case with diseased or inflamed tissue, some tumors, and the capillary beds surrounding the liver and spleen are exceptions and will allow some nanoparticles to pass [48]. While the leakiness of the vascular endothelium allows for more uptake of certain drugs, significant improvements can be made to target nanoparticles to be delivered across the endothelium. Also, leaky vasculature due to diseased or inflamed tissue may actually be less permeable to substances and a higher doses to combat lower permeability have been shown to also decrease the uptake of nanoparticles $[48,54]$. The impermeability of the vascular endothelium remains a problem associated with in vivo drug delivery. Nanoparticle delivery in vivo can see great improvement in passive transport. Presently, passive transport requires extremely high doses of a drug, is slowly dissolved, and is rapidly cleared [48]. Significant improvement can be accomplished by making use of active transport in nanoparticle drug delivery. This can be done through use of heterogeneous caveolae, or invaginations of the plasma membrane, which are involved in endocytosis and transcytosis and can be specifically targeted [55]. The Simian virus 40 is an unusual animal virus that enters the cell through the caveolae which allows the internalized virus to accumulate in the smooth endoplasmic reticulum [56] . The use of active transport, specifically utilizing caveolae, can alleviate problems associated with passive in vivo nanoparticle drug delivery.

\section{The use of an fda biowaiver}

A biowaiver has been regarded as an official approval of the waiver for conducting a bioequivalence study in the context of an application for drug approval process [57]. Bioequivalence is an important parameter in the process of drug development that is needed to be performed when there is a change in the formulation of dosage form [57]. Seldom, in vivo studies may be waived under the guidelines of the Food and Drug Administration when the challenges listed above are not expected to be a problem [20]. This is because in vitro study design has become more and more accurate in imitating in vivo results, as well as when a high in Vitro/in Vivo Correlation (IVIVC) is expected based upon the characteristics of the drug [23]. An extremely rigorous dissolution standard is necessary for the in vivo biowaiver, and in vitro data must support the request of the biowaiver $(23,58)$. The FDA has determined five categories applicable for biowaivers per their Guidance for Industry; the drug and excipients of interest must have data supporting that both are highly soluble and highly permeable and have rapid and similar dissolution as the five categories [20].

\section{Animal Models Used in in vivo Research}

\section{The role of model animals}

The ability of scientists to enhance and improve the well-being of humans and animals depends wholly on advancements made in research by use of animal models [59]. The use of animal models is usually vital for drug absorption, metabolism, distribution, and excretion studies in which animal models are available for the use of testing drug characteristics in vivo. Due to the many physiological differences between humans and animals, even with the close relationship between the human and primate, using a model animal does not allow one to expect completely similar results if the drug testing reaches the clinical trial stage. For example, the bovine stomach is more complex than a human's and also contains different microflora and ranges from $\mathrm{pH}$ of three to six [41]. In fact, clinical trials are essential to understanding a drug's characteristics because animal studies do not predict with sufficient certainty what will happen in humans [60]. It is also the probability of reduced external validity of animal studies when the drug performs in human trials due to the assessment of the drug's effect on homogenous groups of animals versus heterogeneous humans [60]. Even so, drug testing in a model animal is useful in documenting toxicities, adverse reactions, and drug-drug interactions, among pharmacokinetic characteristics, before it reaches human trials to ensure the safety of the drug. 


\section{Choosing a model animal}

The animal model chosen depends heavily on the goal of the research. Animal models may be homologous, isomorphic, or predictive [61]. An ideal animal disease model for target validation would be a model that recapitulates the disease phenotype, shares the same pathophysiology as human, and responds to existing human therapies in a manner similar to patients. Animal models that faithfully resemble the disease pathophysiology are invaluable for the characterization of the mechanism of action, pharmacokinetics, pharmacodynamics, biomarkers, safety, and toxicity of future therapies. Such animal models could also help to predict the human dose prediction for clinical trials [62]. Homologous animal models are identical to human physiology, pathology, and treatment in every way [61]. Isomorphic models resemble the human disorder, but the disease has been induced in some way [61]. Predictive animal models are not identical to the human disorder; these models allow in some way to make predictions or comparisons of the human disease, treatment, and effect of said treatment [61]. Ethics, availability, housing requirements, ease of handling, cost, and susceptibility to disease must also be taken into account when choosing an animal model $[63,64]$.

Both invertebrate and vertebrate models are used in drug testing. Oftentimes, invertebrate animal models are used in drug studies involving neurological, genetic, and developmental disorders [64]. One such invertebrate used with frequency is the zebrafish [65]. This model is used especially when researchers are interested in an embryologically and genetically tractable disease model [66]. Vertebrate models include most large and small animal models, such as baboons, macaques, cows, dogs, rabbits, guinea pigs, rats, and mice which have been traditionally used. These models may be the most important to use in translational research [64].

General principles must be considered when choosing an animal such as high numbers of results and relevancy of life cycle. An invertebrate would make a great choice if high numbers of results are needed; nevertheless, one must consider biological sampling ability and relevancy of life cycle. For example, a pair of zebrafish can produce 100-300 embryos weekly which allows even a small-scale zebrafish facility to produce large numbers of embryos and a high number of results at a reduced cost [67]. Although the zebrafish is gaining popularity as an animal model, the animals used most commonly in drug testing are genetically-modified mice, rats, dogs, and nonhuman primates [68]. Animals should be chosen on the basis of the physiological and biochemical similarities between the animal model and humans and the underlying mechanisms of drug absorption, distribution, metabolism, and excretion in the animal [69]. There are a number of examples of established animal models used for particular diseases (Table 2) [64]. Furthermore, important physiological and biochemical factors used to determine which animal model to select include organ blood flow, blood volume, blood $\mathrm{pH}$, tissue distribution, localization of drug transporters, and localization of metabolizing enzymes as compared to those of humans [69].

Aanimal models used in absorption studies: Absorption characteristics are most influenced by amount and location of drug receptors as well as gastrointestinal $\mathrm{pH}[70,69]$. Accordingly, the dog is the animal model most commonly used for in vivo absorption studies for drugs whose absorption is dependent upon $\mathrm{pH}$ [71]. The dog is known to have similar $\mathrm{pH}$ to humans changes overall, although they have a higher $\mathrm{pH}$ initially which makes them great models for drugs whose absorption may be affected by the fasted or fed state [69]. Additionally, dogs and humans have very similar distribution of multi-drug resistance protein 2 mRNA, which is unquestionably associated with drug resistance [69]. However, the contraction and expulsion times are different between dogs and humans [41]. Also, many drugs have intrinsic effects on gastrointestinal motility and can potentially modify the absorption of other drugs [72]. Some flaws in model animals, in correspondence to other species, may be overlooked if they're not expected to become problematic in the clinical trials.

The rat and other rodents are other animals are commonly used for investigating $\mathrm{pH}$-sensitive drugs. The gastrointestinal $\mathrm{pH}$ and changes in $\mathrm{pH}$ are not very comparable to those of humans, unlike the dog [69]. The similarity of drug transporters allows oral drug absorption, but not oral bioavailability, to be predicted with great success in the rat model [69]. The rat shares the same expression and distribution of drug transporters in the intestine with the exception of P-glycoprotein,

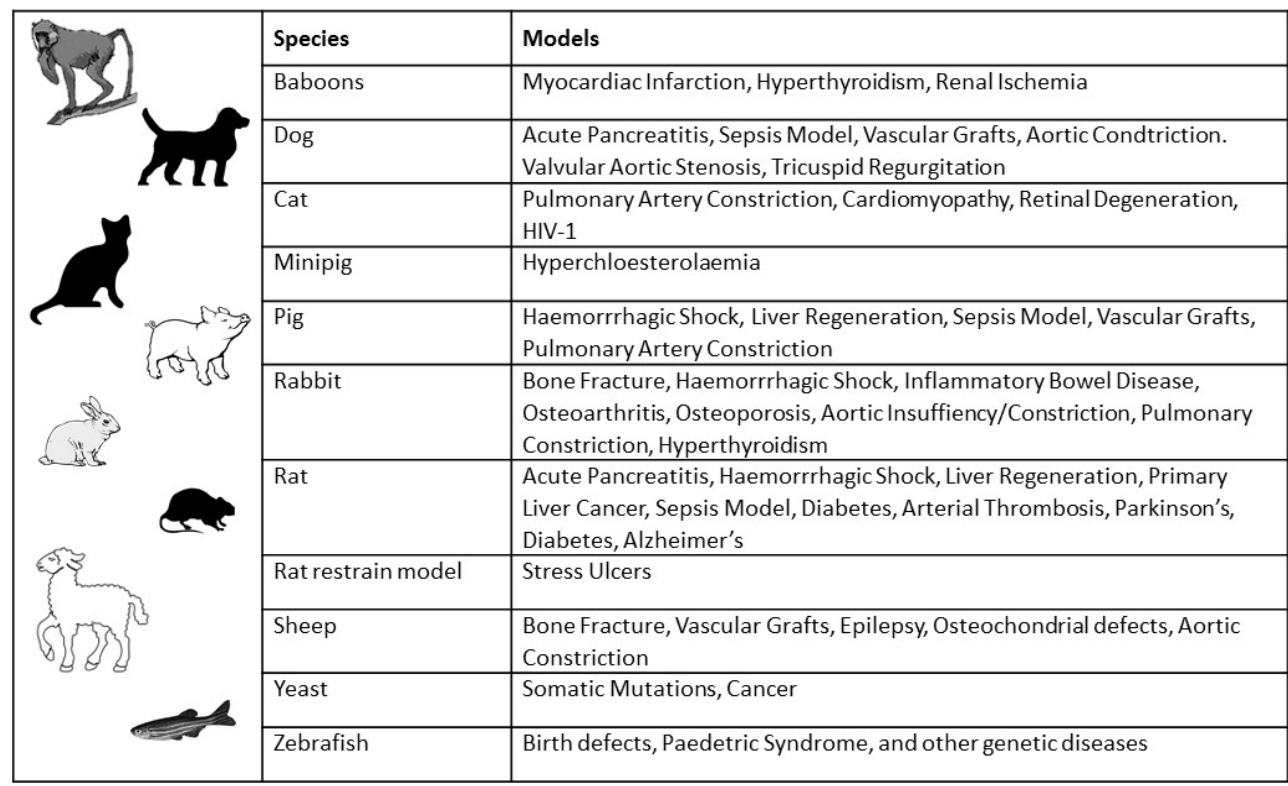

Table 2: Some animal species have been ingrained as models for specific diseases and areas of study $[4,8,10,64,66,10,104-115]$. 
multi-drug resistance protein 3 , and glucose transporter 1 and 3 [69]. Unlike the rat, the rabbit is the only laboratory rodent used in absorption and permeability studies using the buccal mucosa route that has a non-keratinized mucosal lining similar to human tissue and is used extensively in experimental studies [73].

Current data suggests that no single animal can mimic the gastrointestinal characteristics of a human [74]. However, non-human primates are the closest in similar characteristics to humans in their anatomy and physiology of the gastrointestinal tract. Gastric $\mathrm{pH}$, gastric emptying time, contraction intensity, and small intestine transit time are all comparable to those in humans [75]. One complication with using a non-human primate is that some monkeys have significantly higher first-pass metabolism and higher levels of CYP3A subfamily enzyme, multi-drug resistance protein 1 and 2, and breast cancer resistance protein [69]. Another animal model should be used for absorption and metabolic studies if the drug of interest is expected to be metabolized by these enzymes.

Animal models used in distribution studies: Since the major determinant of drug distribution is based on carrier-mediated drug transporters in the liver and the degree of efflux through the canalicular membrane, rats and mice are the most common animal models used for distribution studies [69]. The use of organic anion transport polypeptides (OATPs) in rodents resembles the use of OATPs in humans, and even information on drug-drug interaction can be discovered through use of rodents and applied to humans [69]. For example, rifampin's inhibition of hepatic uptake by OATP1a4 decreases the volume of distribution similarly in both humans and rodents [76]. Even early in the discovery process, pharmacokinetic screening using various dose regimens and administration routes in rodents or allows rapid eliminations of drug candidates, which facilitates the production of a candidate that is more likely to succeed in preclinical testing [77].

Animal models used in metabolism studies: Metabolism studies tend to focus on changes in activity or expression of metabolizing enzymes. In vivo metabolism studies most commonly emphasize the Cytochrome P450 family in the liver which is specifically true for study involving drug-drug interactions [69]. Since different cytochrome P450 enzymes can catalyze the same activity, additional information is required to determine the animal species most similar to man with regard to the various cytochrome P450 activities [78]. Higher animals, such as cynomolgus monkeys, rhesus monkeys, and beagle dogs have the most similar association between enzyme kinetics and activities [69]. Rodents as a model animal in metabolism studies are limited because much information about the kinetics of their cytochrome enzyme activity and various drugs' effects on said enzymes remains unknown [79]. Rats also have different isoforms of CYP enzymes that are not found in humans, causing predictions based on data more difficult [69].

Rhesus and cynomolgus monkeys possess the most metabolic similarities related to humans, specifically regarding the CYP enzymes [80]. Eleven members of CYP1A, CYP2A, CYP2C, CYP2D, CYP2E, and CYP3A subfamilies are at least $90 \%$ homologous in amino acid sequences [69]. The CYP2C76 is only approximately $70 \%$ homologous to humans, which may account for differences in metabolism of some drugs [69]. This underlines the importance of understanding the metabolism of a drug when choosing an animal model.

Animal models used in excretion studies: The rate of renal excretion is determine by renal blood flow, glomerular filtration rate
(GFR), and tubular secretion and reabsorption [81]. Because the GFR is associated with the number of nephrons, it varies widely throughout the available animal models [69]. Any species that uses GFR and passive reabsorption as the major mechanism for renal excretion would make a good animal model for an in vivo excretion study [71]. Nevertheless, the rate and amount of drugs that are excreted by tubular secretion and reabsorption differ across various animal models [69]. The FDA encourages the identification of differences in drug metabolism between animals used in nonclinical safety assessments and humans as early as possible during the drug development process [82]. In order to apply the data received from animal model excretion studies, the dose should be adjusted appropriately depending on the rate of renal excretion and the number of nephrons.

The principle transporters for renal uptake in the renal tubules are the enzymes in the family of organic cationic transporters (OCTs) and organic anionic transporters (OATs) [69]. Different forms of OCTs and OATs are found in different species. For example, in humans, OCT2 and OAT2 are the most common forms with OCT2 considered as a kidney transporter and OAT2 identified at the basolateral membrane of proximal tubules [83]. In rats, OCT1, OCT2, OAT1, and OAT2 are the main enzymes involved in renal metabolism [69]. A favorable correlation exists for OAT1 in humans, cynomolgus monkeys, and rats whereas OAT3 correlation exists only for humans and cynomolgus monkeys [69]. The choice of animal model should reflect similarities between the animal and humans in their transport enzymes if the drug will be excreted renally.

\section{In vivo Research Techniques}

As previously stated, the metabolism and drug-drug interactions must be made known before any drug is approve. As of current practices, there is no one single study that is correct for determining the characteristics of a drug in vivo [84]. There are many routes and sampling techniques available for analyzing specific in vivo features of a drug action. Drug administration to the model animal include oral, transdermal, ocular, subcutaneous, intramuscular, and intravenous among other routes of delivery [85]. Early knowledge of tissue distribution of drugs and their metabolites is extremely important in understanding pharmacological responses [pharmacokinetics (PK), pharmacodynamics (PD), drug transport, toxicity], and in predicting undesirable off-target effects (safety, drug-drug interactions). While the physico-chemical properties of a compound (membrane permeability, protein binding, lipophilicity etc.) can be measured and modelled to predict biodistribution, tissue exposure has historically been inferred from surrogate measures such as concentrations of drug in plasma or tissue homogenates [86]. While such methods enable high throughput screening during the discovery phase, reliance on circulating concentrations can prove erroneous when assessing tumor or blood-brain barrier penetration or highly localized delivery within multi-cellular tissues. Therefore techniques allowing histological assignment of drug distribution within tissue are required [86]. The measurement of distribution for the drug of interest can be accomplished through many techniques such as equilibrium dialysis, isolated lung perfusion, microdialysis, and imaging techniques. Newer techniques like microdialysis, positron emission tomography (PET) and magnetic resonance spectroscopy (MRS) offers many advantages compared to conventional techniques such as tissue biopsy, skin blister fluid sampling, saliva sampling etc. These advantages include technique being semi-invasive, direct concentration measurement, measurement at multiple sites, continuous monitoring, low technical complexity and cost [87]. 


\section{Equilibrium dialysis}

Equilibrium dialysis is used to determine the amount of a ligand bound to a macromolecule using tissue homogenate [34]. This was initially used to measure the amount of plasma protein-binding, but has since been modified for its use in measuring drugs binding to tissue proteins [34]. Usually dialysis is performed between tissue homogenates and whole blood, allowing the drug to cross through the dialysis membrane [34]. This method and similar dialysis experiments will reveal distribution, but clarify little about the localization of the drug [34]. Although there is no standard in measuring protein binding in vitro, equilibrium dialysis is often regarded as the reference method for determining a drug's protein binding characteristics [88].

\section{Isolated organ perfusion}

Isolated organ perfusion involves maintaining an organ in viable status, either by single pass or recirculation with the medium. Single pass is used in experiments investigating distribution while recirculation is associated with metabolism and excretion studies. Isolated organ perfusion has been documented in distribution studies involving several organs, including lung, kidney, and brain [34]. Viability of the organ is crucial for this technique. Traditionally, viability has been obtained through use of hypothermia and exposure to nutrient solutions. Currently, the organs are kept viable through constant and controlled perfusion pressure or perfusate flow [89]. The lung was identified as an ideal organ for isolated perfusion because of its symmetry, an exclusive arterial supply from the pulmonary artery, venous drainage into 2 pulmonary veins, and tolerance for hyperthermic conditions without significantly impairing systemic function. Isolated lung perfusion (ILP) is a surgical technique developed to deliver highdose chemotherapy to the lung. This technique minimizes exposure by selectively delivering the agent through the pulmonary artery and selectively diverting venous effluent, which is advantageous in limiting exposure to critical organs and minimizing the impact of active drug loss from renal metabolism [90]. Another organ that is used for isolated perfusion is the liver. Isolated hepatic perfusion is a surgical technique used for treatment of nonresectable liver cancer, liver metastases, and melanoma where systemic chemotherapy is the only other option. The isolation of the liver allows for a delivery of substantially higher doses of chemotherapy at elevated temperatures that would be lethal if administered by traditional systemic delivery [91,92]. These isolated organ perfusion techniques allow higher concentrations of chemotherapy to be delivered to the targeted organ while ensuring the vitality of other organs.

\section{Microdialysis}

Microdialysis offers significant advantages to determining the protein bonding in vivo and is one of the most preferred methods of quantifying the pharmacokinetics of a drug [88]. This technique is especially useful in explanation of drug distribution and receptor phase pharmacokinetics [87]. It is a powerful sampling technique wherein regional chemical (or biochemical) information regarding is obtained by implantation of a semi-permeable membrane into virtually any tissue of interest (brain, blood, bile, eye, etc.) [92]. Microdialysis is performed by the use of a probe connected to a tube attached to a dialysis membrane that allows fluid circulation, imitating the solution (termed perfusate) of similar ionic strength and $\mathrm{pH}$ as the surrounding fluid, tissue, blood capillary [34]. This method is semi-invasive due to the probe needing constant perfusion [87]. However, the invasiveness is minimal due to the small sample volumes which are usually measured in just microliters [87]. The perfusate collected is analyzed chemically and the results reflect the composition of the fluid with time due to the diffusion of substances back and forth over the membrane [93]. This is advantageous in determining the chronological data of a distribution study [34]. Microdialysis allows the simultaneous determination of different physiological parameters such as blood pressure, locomotor and convulsive activity, which renders it a suitable tool for pharmacokinetic-pharmacodynamic studies of drugs and modeling. The reverse microdialysis is a powerful and effective technique in the study of local actions of drugs to different tissues such as specific brain nuclei, liver, or skeletal muscle [94,95]. Benefits that are offered with microdialysis include that since tissues and fluids do not have to be removed or sacrificed, none of the pharmacokinetic attributes are changed by the sampling, several samples may be collected with ease, and lower costs $[34,87]$.

\section{Imaging techniques}

Imaging techniques are beneficial, non-invasive tools to to elucidate and demonstrate the mechanistic actions of drugs in vivo [87]. Imaging techniques that are frequently used for in vivo drug distribution measurements include autoradiography, magnetic resonance imaging (MRI), magnetic resonance spectroscopy (MRS), and positron emission tomography (PET). They are especially useful with organ and tissue sites that can only be reached by surgical methods, such as the brain, where microdialysis cannot be used [87]. Since imaging methods are non-invasive, they allow for longitudinal studies in a single animal, therefore increasing the statistical relevance of a study [95].

Autoradiography: Autoradiography uses radio-labeled isotopes in the tissue of interest to image through photographic emulsion [34]. This technique was used mostly in the past at the tissue or cellular levels, but can now be brought to the subcellular level [96]. Autoradiography is advantageous because it allows mapping of drug distribution within the body. Be that as it may, a high number of animals are necessary for data collection for autoradiography [34].

Magnetic resonance imaging and magnetic resonance spectroscopy: Magnetic resonance imaging and magnetic resonance spectroscopy are two very frequently used methods to determine drug distribution. MRIs employ radio frequency pulses and magnetic fields to find signals from changes in nuclear magnetic moments [87] that can be measured when certain biologically important nuclei, such as $1 \mathrm{H}, 13 \mathrm{C}$ and $31 \mathrm{P}$ are placed in a high magnetic field. As many of these nuclei form an essential part of the biological systems, being building blocks for water and organic molecules, MRI signal can be measured without any external tracers or radioactive irradiation. As MRI signal is in the radiofrequency (RF) part of the electromagnetic spectrum, it has excellent tissue penetration and minimal interaction with tissue which makes MRI a non-invasive and safe imaging technique [97]. MRS uses the same principles as MRI, but is more specific to allow for better characterization of drug distribution and is used to detect drug metabolites in addition to un-metabolized drug [87]. Magnetic resonance spectroscopy complements magnetic resonance imaging as a non-invasive means for the characterization of tissue [98]. One setback with both MRI and MRS is that drug must be present in a somewhathigh concentration to be able to be detected in vivo [87].

Positron emission tomography (PET): PET makes use of positron-emitting radioisotopes that pass through a tissue and release photons in opposite directions [87]. This technique is able to propose three-dimensional images with great spatial resolution. PET is another imaging technique that is non-invasive that is useful in tissue drug distribution information, although it is not implemented as frequently 
as MRI and MRS. However, it is very favorable for drugs directed at the cerebral nervous system, because of the ability to confirm exposure to the brain [99].

Neuroimaging techniques allow the provision of detailed structural, metabolic, and functional information in vivo on the human/animal brain, thus, making a significant contribution to our understanding of drug-effects on brain systems. MRI and PET provides a direct way of investigating the pathophysiology of disease in vivo diagnosis and monitor the neuroscientific investigation of drug action mechanisms and provide quantitative markers of drug action, or endpoints, in candidate compounds for the clinic [100]. Epilepsy, brain tumors, Parkinson's diseases, schizophrenia, and Alzheimer's dieases are common neurologic disorders and the neuroimaging modalities mentioned above are extensively utilized to identify early biomarkers involved in these diseases, longitudinally monitor disease progression, and assess the effectiveness of therapies [97,100-102]. PET has a high translational applicability from rodents to man and measures total drug concentrations in vivo for studying neuropharmacokinetics. In vivo estimation of drug blood-brain barrier transport and brain unbound concentrations of opioid agonist oxycodone and its neuro pharmacokinetic rats was investigated by correlating the combined PET and microdialysis [103-115].

\section{Conclusion}

In vivo studies are crucial in drug development for evaluating a drug's pharmacokinetic and pharmacodynamics characteristics. They are essential because in vitro studies do not possess the ability to provide quantitative results of absorption, distribution, metabolism, and excretion in animal and human models. An animal model should be considered on the basis of the physiological and biochemical similarities between the animal model and humans in addition to the underlying mechanisms of drug absorption, distribution, metabolism, and excretion in the animal. Many semi-invasive and non-invasive techniques, such as MRI and microdialysis, have recently replaced older techniques, such as skin blistering, for collecting in vivo data. However, much work is still needed for the advancement of animal study data to better resemble human clinical trials.

\section{References}

1. Csermely P, Korcsmáros T, Kiss H J, London G, Nussinov R (2013) Structure and dynamics of molecular networks: A novel paradigm of drug discovery: a comprehensive review. Pharmacology \& Therapeutics, 138: 333-408.

2. FDA (2017) The Drug Development Process

3. Hughes JP, Rees S, Kalindjian SB, Philpott K L (2011) Principles of early drug discovery. British Journal of Pharmacology 162: 1239-1249.

4. Kurz K, Main B, Sandusky GE (1990) Rat model of arterial thrombosis induced by ferric chloride. Thrombosis Research 60: 269-280.

5. Allen L, Ansel HC (2013) Ansel's pharmaceutical dosage forms and drug delivery systems: Lippincott Williams \& Wilkins.

6. Haber VE, Spaventi R (2017) Discovery and Development of Novel Drugs Blue Biotechnology. Springer. pp: 91-104.

7. Drug Discovery and Development, Understanding the R\&D Process (2007) innovationorg. Pharmaceutical Research and Manufacturers of America

8. Björklund LM, Sánchez-Pernaute R, Chung S, Andersson T, Chen IYC, et al (2002) Embryonic stem cells develop into functional dopaminergic neurons after transplantation in a Parkinson rat model. Proceedings of the National Academy of Sciences 99: 2344-2349.

9. Lipsky MS, Sharp LK (2001) From idea to market: the drug approval process The Journal of the American Board of Family Practice 14: 362-367.

10. Martini L, Fini M, Giavaresi G, Giardino R (2001) Sheep model in orthopedic research. A Literature Review Comparative Medicine 51: 292-299.
11. Pandey S, Pandey P, Tiwari G, Tiwari R (2010) Bioanalysis in drug discovery and development. Pharmaceutical Methods 1: 14-24.

12. Thompson TN (2000) Early ADME in support of drug discovery: The role of metabolic stability studies. Current Drug Metabolism 1: 215-241.

13. Baranczewski P, Stanczak A, Sundberg K, Svensson R, Wallin A, et al. (2006) Introduction to in vitro estimation of metabolic stability and drug interactions of new chemical entities in drug discovery and development. Pharmacological Reports. 58: p453.

14. Andrade E, Bento A, Cavalli J, Oliveira S, Freitas C, et al. (2016) Non-clinica studies required for new drug development-Part I: Early in silico and in vitro studies, new target discovery and validation, proof of principles and robustness of animal studies. Brazilian Journal of Medical and Biological Research 49:e5644

15. Polli, JE (2008) In vitro studies are sometimes better than conventional human pharmacokinetic in vivo studies in assessing bioequivalence of immediaterelease solid oral dosage forms. The AAPS Journal. 10: 289-299.

16. Bowes J, Brown AJ, Hamon J, Jarolimek W, Sridhar A, et al. (2012) Reducing safety-related drug attrition: The use of in vitro pharmacological profiling Nature Reviews Drug Discovery 11: 909.

17. Zhou W, Wang Y, Lu A, Zhang G (2016) Systems pharmacology in smal molecular drug discovery. International Journal Of Molecular Sciences. 17: 246

18. National Research Council (1999) A Report of the Committee on Methods of Producing Monoclonal Antibodies Institute for Laboratory Animal Research National Research Council: National Academy Press, Washington, DC.

19. Council NR (2010) Guide for the care and use of laboratory animals: National Academies Press.

20. FDA (2015) Waiver of in vivo Bioavailability and Bioequivalence Studies for Immediate-Release Solid Oral Dosage Forms Based on a Biopharmaceutics Classification System.

21. Nainar S, Rajiah K, Angamuthu S, Prabakaran D, Kasibhatta R (2012) Biopharmaceutical classification system in in vitro/in vivo correlation: Concept and development strategies in drug delivery tropical. Journal of Pharmaceutical Research 11: 319-329.

22. Sewell F, Aggarwal M, Bachler G, Broadmeadow A, Gellatly N, et al. (2017) The current status of exposure-driven approaches for chemical safety assessment: a cross-sector perspective. Toxicology 389: 109-117.

23. http://wwwhc-scgcca/dhp-mps/brgtherap/applic-demande/guides/semensperme-acces/dssap-passd_gui_doc-ori-engphp

24. Singhvi G, Singh M (2011) Review: in vitro drug release characterization models. Int J Pharm Stud Res 2: 77-84.

25. Stegemann S, Leveiller F, Franchi D, De-Jong H, Lindén H (2007) When poor solubility becomes an issue: From early stage to proof of concept. European Journal of Pharmaceutical Sciences 31: 249-261.

26. http://www.particlesciences.com/news/technical-briefs/2010/in-vitrodissolution-testing-for-solid-oral-dosage-forms.html

27. Emami J (2006) In vitro-in vivo correlation: From theory to applications. J Pharm Pharm Sci 9: 169-189.

28. Bamigbola EA (2012) Correlation of in vitro dissolution profiles with in vivo pharmacokinetic parameters of some commercial brands of aspirin tablets marketed in Nigeria Readings in Advanced Pharmacokinetics-Theory, Methods and Applications: InTech.

29. Ghosh A, Choudhury GK (2009) In vitro-in vivo correlation (IVIVC): a review Journal of Pharmacy Research. 2: 1255-1260.

30. Amoedo N, Obre E, Rossignol R (2017) Drug discovery strategies in the field of tumor energy metabolism: Limitations by metabolic flexibility and metabolic resistance to chemotherapy Biochimica et Biophysica Acta (BBA)Bioenergetics 1858: 674-685

31. Brajša K, Vujasinović I, Jelić D, Trzun M, Zlatar I, et al. (2015) Antitumor activity of amidino-substituted benzimidazole and benzimidazo [1, 2-a] quinoline derivatives tested in $2 \mathrm{D}$ and $3 \mathrm{D}$ cell culture systems. Journal of Enzyme Inhibition and Medicinal Chemistry 31: 1139-1145.

32. Blomme EA, Will Y (2015) Toxicology strategies for drug discovery: present and future. Chemical Research in Toxicology. 29: 473-504. 
Citation: Brake K, Gumireddy A, Tiwari A, Chauhan H, Kumari D (2017) In vivo Studies for Drug Development via Oral Delivery: Challenges, Animal Models and Techniques. Pharm Anal Acta 8: 560. doi: 10.4172/2153-2435.1000560

33. Cardot J, Beyssac E, Alric M (2007) In vitro-in vivo correlation: Importance of dissolution in IVIVC. Dissolution Technologies 14: p15.

34. Lanao J, Fraile M (2005) Drug tissue distribution: Study methods and therapeutic implications. Current Pharmaceutical Design 11: 3829-3845.

35. Sjögren E, Abrahamsson B, Augustijns P, Becker D, Bolger MB, et al. (2014) In vivo methods for drug absorption: Comparative physiologies, model selection, correlations with in vitro methods (IVIVC), and applications for formulation/ $\mathrm{API} /$ excipient characterization including food effects. European Journal of Pharmaceutical Sciences 57: 99-151.

36. Ding C, Li Z (2017) A review of drug release mechanisms from nanocarrier systems. Mater Sci Eng C Mater Biol Appl 76: 1440-1453.

37. Pridgen EM, Alexis F, Farokhzad OC (2015) Polymeric nanoparticle drug delivery technologies for oral delivery applications. Expert opinion on drug delivery 12: 1459-1473.

38. Rabanel J, Aoun V, Elkin I, Mokhtar M, Hildgen P (2012) Drug-loaded nanocarriers: Passive targeting and crossing of biological barriers. Current Medicinal Chemistry 19: 3070-3102.

39. Ensign LM, Cone R, Hanes J (2012) Oral drug delivery with polymeric nanoparticles: The gastrointestinal mucus barriers. Advanced Drug Delivery Reviews 64: 557-570.

40. Moroz E, Matoori S, Leroux JC (2016) Oral delivery of macromolecular drugs: Where we are after almost 100 years of attempts. Advanced drug delivery reviews 101: 108-121.

41. Grassi M, Grassi G, Lapasin R, Colombo I (2006) Understanding drug release and absorption mechanisms: A physical and mathematical approach: CRC Press.

42. Won SC, Oberlies HN, Paine FM (2010) Influence of dietary substances on intestinal drug metabolism and transport. Current Drug Metabolism. 11: 778792.

43. Melander A (1978) Influence of food on the bioavailability of drugs. Clinical Pharmacokinetics. 3: 337-351.

44. Tannergren C, Bergendal A, Lennernäs H, Abrahamsson B (2009) Toward an increased understanding of the barriers to colonic drug absorption in humans: Implications for early controlled release candidate assessment. Molecular Pharmaceutics 6: 60-73.

45. Lennernäs H (2013) Human in vivo regional intestinal permeability: importance for pharmaceutical drug development. Molecular pharmaceutics 11: 12-23.

46. Laksitorini M, Prasasty VD, Kiptoo PK, Siahaan TJ (2014) Pathways and progress in improving drug delivery through the intestinal mucosa and bloodbrain barriers. Therapeutic Delivery 5: 1143-1163.

47. Stamatovic SM, Keep RF, Andjelkovic AV (2008) Brain endothelial cell-cel junctions: How to "open" the blood brain barrier. Current Neuropharmacology 6: 179-192.

48. Chrastina A, Massey KA, Schnitzer JE (2011) Overcoming in vivo barriers to targeted nanodelivery. Wiley Interdisciplinary Reviews. Nanomedicine and Nanobiotechnology 3: 421-437.

49. Gaur U, Sahoo SK, De Tapas K, Ghosh PC, Maitra A, Ghosh P (2000) Biodistribution of fluoresceinated dextran using novel nanoparticles evading reticuloendothelial system. International Journal of Pharmaceutics 202: 1-10.

50. Owens DE, Peppas NA (2006) Opsonization, biodistribution, and pharmacokinetics of polymeric nanoparticles. International Journal of Pharmaceutics 307: 93-102.

51. Barua S, Mitragotri S (2014) Challenges associated with penetration of nanoparticles across cell and tissue barriers: A review of current status and future prospects. Nano Today 9: 223-243.

52. Dobrovolskaia MA, Aggarwal P, Hall JB, McNeil SE (2008) Preclinical studies to understand nanoparticle interaction with the immune system and its potential effects on nanoparticle biodistribution. Molecular Pharmaceutics 5: 487-495.

53. Patel HM, Tużel NS, Ryman BE (1983) Inhibitory effect of cholesterol on the uptake of liposomes by liver and spleen. Biochimica et Biophysica Acta (BBA)General Subjects 761: 142-151.

54. Panyam J, Labhasetwar V (2003) Biodegradable nanoparticles for drug and gene delivery to cells and tissue. Advanced Drug Delivery Reviews 55: 329 347
55. Mclntosh DP, Tan XY, Oh P, Schnitzer JE (2002) Targeting endothelium and its dynamic caveolae for tissue-specific transcytosis in vivo: A pathway to overcome cell barriers to drug and gene delivery. Proceedings of the National Academy of Sciences 99: 1996-2001.

56. Pelkmans L, Kartenbeck J, Helenius A (2001) Caveolar endocytosis of simian virus 40 reveals a new two-step vesicular-transport pathway to the ER. 3: 473-483.

57. Rohilla S (2012) Biowaivers Criteria and Requirements. International Journal of Pharmaceutical \& Biological Archive 3: :727-731.

58. Sirisuth N, Eddington ND (2002) The influence of first pass metabolism on the development and validation of an IVIVC for metoprolol extended release tablets. European Journal of Pharmaceutics and Biopharmaceutics 53: 301-309.

59. https://grants.nih.gov/grants/olaw/guide-for-the-care-and-use-of-laboratoryanimals.pdf

60. Van der Worp H B, Howells DW, Sena ES, Porritt MJ, Rewell S, et al. (2010) Can animal models of disease reliably inform human studies? PLoS med 7 : e1000245.

61. Davidson M, Lindsey J, Davis J (1987) Requirements and selection of an animal model. Israel Journal of Medical Sciences 23: 551-555.

62. Sim DS, Kauser K (2015) In vivo target validation using biological molecules in drug development. Handb Exp Pharmacol 232: 59-70.

63. https://www.crcpress.com/Animal-Models-in-Orthopaedic-Research/AnFreidman/p/book/9780849321153

64. http://www.worldscientific.com/worldscibooks/10.1142/6454

65. Zon LI, Peterson RT (2005) In vivo drug discovery in the zebrafish. Nature Reviews Drug Discovery 4: 35-44

66. Lieschke GJ, Currie PD (2007) Animal models of human disease: Zebrafish swim into view. Nature Reviews Genetics 8: 353-367.

67. Kari G, Rodeck U, Dicker AP (2007) Zebrafish: An emerging model system for human disease and drug discovery. Clinical Pharmacology \& Therapeutics 82: $70-80$.

68. NCO (2005) The Ethics of Research Involving Animals London. Nuffield Council on Bioethics: 376.

69. Tang C, Prueksaritanont T (2010) Use of in vivo animal models to assess pharmacokinetic drug-drug interactions. Pharmaceutical research 27: 1772-1787.

70. Sakai JB (2009) Practical pharmacology for the pharmacy technician.

71. Lin JH (1995) Species similarities and differences in pharmacokinetics. Drug Metabolism and Disposition 23: 1008-1021.

72. Prescott L (1974) Gastric emptying and drug absorption. British Journal of Clinical Pharmacology 1: 189-190.

73. Shojaei AH (1998) Buccal mucosa as a route for systemic drug delivery: A review. J Pharm Pharm Sci 1: 15-30.

74. Kararli TT (1995) Comparison of the gastrointestinal anatomy, physiology, and biochemistry of humans and commonly used laboratory animals. Biopharmaceutics \& Drug Disposition 16:351-380.

75. Ikegami K, Tagawa K, Narisawa S, Osawa T (2003) Suitability of the cynomolgus monkey as an animal model for drug absorption studies of ora dosage forms from the viewpoint of gastrointestinal physiology. Biological and Pharmaceutical Bulletin 26: 1442-1447.

76. Lam JL, Shugarts SB, Okochi H, Benet LZ (2006) Elucidating the effect of final-day dosing of rifampin in induction studies on hepatic drug disposition and metabolism. Journal of Pharmacology and Experimental Therapeutics 319 : 864-870.

77. Zhang D, Luo G, Ding X, Lu, C (2012) Preclinical experimental models of drug metabolism and disposition in drug discovery and development. Acta Pharmaceutica Sinica B 2: 549-561.

78. Guengerich FP (1997) Comparisons of catalytic selectivity of cytochrome P450 subfamily enzymes from different species. Chemico-Biological Interactions 106: $161-182$.

79. Gibson G, Plant N, Swales K, Ayrton A, El-Sankary W (2002) Receptordependent transcriptional activation of cytochrome P4503A genes: Induction mechanisms, species differences and interindividual variation in man Xenobiotica 32: 165-206. 
Citation: Brake K, Gumireddy A, Tiwari A, Chauhan H, Kumari D (2017) In vivo Studies for Drug Development via Oral Delivery: Challenges, Animal Models and Techniques. Pharm Anal Acta 8: 560. doi: 10.4172/2153-2435.1000560

Page 11 of 11

80. Iwasaki K, Uno Y (2009) Cynomolgus monkey CYPs: A comparison with human CYPs. Xenobiotica 39: 578-581.

81. Stevens LA, Coresh J, Greene T, Levey A S (2006) Assessing kidney function: Measured and estimated glomerular filtration rate. New England Journal of Medicine 354: 2473-2483.

82. http://www.sgs.pt/ /media/Global/Documents/Technical\%20Documents/SGS Clinical-14C-ADME-Clinical-Trials-EN-09.pdf

83. Roth M, Obaidat A, Hagenbuch B (2012) OATPs, OATs and OCTs: The organic anion and cation transporters of the SLCO and SLC22A gene superfamilies. British Journal of Pharmacology 165: 1260-1287

84. h t t p : / / w w w f d a g o v / d o w n I o a d s / D r u g s GuidanceComplianceRegulatoryInformation/Guidances/ucm072119pdf

85. Cheng Y, Xu Z, Ma M, Xu T (2008) Dendrimers as drug carriers: Applications in different routes of drug administration. Journal of Pharmaceutical Sciences 97: 123-143.

86. Cobice D, Goodwin R, Andrén PE, Nilsson A, Mackay C, et al. (2015) Future technology insight: Mass spectrometry imaging as a tool in drug research and development. British Journal of Pharmacology 172: 3266-3283.

87. Brunner M, Langer O (2006) Microdialysis versus other techniques for the clinical assessment of in vivo tissue drug distribution. The AAPS Journal 8: E263-E271.

88. Zeitlinger MA, Derendorf H, Mouton JW, Cars O, Craig WA, et al. (2011) Protein binding: Do we ever learn? Antimicrobial Agents and Chemotherapy 55: 3067-3074.

89. http://www.google.co.in/patents/US5338662

90. Cypel M, Keshavjee S (2016) Novel Technologies for Isolated Lung Perfusion. Thoracic Surgery Clinics 26: 139-145.

91. Reddy SK, Kesmodel SB, Alexander Jr HR (2014) Isolated hepatic perfusion for patients with liver metastases. Therapeutic Advances in Medical Oncology 6: 180-194.

92. Weiss DJ, Lunte CE, Lunte SM (2000) In vivo microdialysis as a tool for monitoring pharmacokinetics TrAC. Trends in Analytical Chemistry 19: 606616

93. Ungerstedt U (1991) Microdialysis: Principles and applications for studies in animals and man. Journal of internal Medicine 230: 365-373.

94. Hocht C, Opezzo JA, Taira CA (2004) Microdialysis in drug discovery. Current Drug Discovery Technologies 1: 269-285.

95. Rudin M, Weissleder R (2003) Molecular imaging in drug discovery and development. Nature Reviews Drug Discovery 2: 123-131.

96. Caro LG, Van Tubergen RP (1962) High-resolution autoradiography I Methods. The Journal of Cell Biology 15:173-188.

97. Bertoglio D, Verhaeghe J, Dedeurwaerdere S, Gröhn O (2017) Neuroimaging in animal models of Epilepsy neuroscience. Neuroscience 358: 277-299.

98. Gujar SK, Maheshwari S, Björkman-Burtscher I, Sundgren PC (2005) Magnetic resonance spectroscopy. Journal of Neuro-Ophthalmology 25: 217-226.
99. Lee C-M, Farde L (2006) Using positron emission tomography to facilitate CNS drug development. Trends in Pharmacological Sciences 27: 310-316.

100. Wise RG, Tracey I (2006) The role of fMRI in drug discovery. Journal of Magnetic Resonance Imaging 23: 862-876.

101. McGuire P, Howes OD, Stone J, Fusar-Poli P (2008) Functional neuroimaging in schizophrenia: Diagnosis and drug discovery. Trends in Pharmacological Sciences 29: 91-98

102.Zhao Y, Raichle ME, Wen J, Benzinger TL, Fagan AM et al. (2017) In vivo detection of microstructural correlates of brain pathology in preclinical and early Alzheimer Disease with magnetic resonance imaging. Neurolmage 148 296-304.

103. Gustafsson S, Eriksson J, Syvänen S, Eriksson O, Hammarlund-Udenaes M, et al. (2017) Combined PET and microdialysis for in vivo estimation of drug blood-brain barrier transport and brain unbound concentrations. Neurolmage 155: $177-186$

104. Hartwell LH (2004) Yeast and cancer. Bioscience Reports 24: 523-544.

105. Reed M, Meszaros K, Entes L, Claypool M, Pinkett J, et al. (2000) A new rat model of type 2 diabetes: The fat-fed, streptozotocin-treated rat. Metabolism 49: $1390-1394$

106. Johnston SC, Horn JK, Valente J, Simon RP (1995) The role of hypoventilation in a sheep model of epileptic sudden death. Annals of Neurology 37: 531-537.

107.Kandel R, Grynpas M, Pilliar R, Lee J, Wang J, et al. (2006) Repair of osteochondral defects with biphasic cartilage-calcium polyphosphate constructs in a sheep model. Biomaterials 27: 4120-4131.

108. LaFerla FM, Green KN (2012) Animal models of Alzheimer disease. Cold Spring Harbor Perspectives in Medicine 2: a006320.

109. Hasenfuss G (1998) Animal models of human cardiovascular disease. Heart failure and hypertrophy Cardiovascular Research 39: 60-76.

110. Tilley L, Liu S, Gilbertson S, Wagner B, Lord P (1977) Primary myocardia disease in the cat $A$ model for human cardiomyopathy. The American Journal of Pathology 86: 493.

111. Menotti-Raymond $M$, David VA, Schäffer AA, Stephens, $R$ Wells $D$, et al. (2007) Mutation in CEP290 discovered for cat model of human retinal degeneration. Journal of Heredity 98: 211-220.

112. Phillips TR, Billaud J, Henriksen S (2000) Methamphetamine and HIV 1: potential interactions and the use of the FIV/cat model. Journal of Psychopharmacology 14: 244-250.

113. Mauser PJ, Pitman A, Witt A, Fernandez X, Zurcher J, et al. (1993) Inhibitory effect of the TRFK-5 anti-IL-5 antibody in a guinea pig model of asthma. American Review of Respiratory Disease 148: 1623-1627.

114. Blander SJ, Horwitz MA (1993) Major cytoplasmic membrane protein of Legionella pneumophila, a genus common antigen and member of the hsp 60 family of heat shock proteins, induces protective immunity in a guinea pig model of Legionnaires' disease. Journal of Clinical Investigation 91: 717-723.

115. Pal PG, Horwitz MA (1992) Immunization with extracellular proteins of Mycobacterium tuberculosis induces cell-mediated immune responses and substantial protective immunity in a guinea pig model of pulmonary tuberculosis. Infection and Immunity 60: 4781-4792. 